diameter from 15 to $90 \mathrm{~mm}$. 17 cases showed spontaneous regression up to a complete remission, 13 of them measuring $<50 \mathrm{~mm}$, while all other cases were managed surgically with a laparoscopic approach. When ovarian parenchyma was macroscopically evident it has been preserved. In 4 cases pathologic examination showed absence of any trace of ovarian parenchyma.

Results 11 cysts, all showing associated fluid corpuscular content were treated surgically, 8 of them larger than $50 \mathrm{~mm}$ and. Among these, 5 presented torsion and one auto-amputation. In one case anaphylactic shock occurred during surgery. In all cases in which ovarian parenchyma was preserved, US follow-up at three months after surgery showed vital ovarian tissue.

Conclusions Ovarian cysts with size exceeding $50 \mathrm{~mm}$ are related to high risk of torsion. Cysts with corpuscular content show almost no tendency to regression after depletion of maternal hormones stimulation: surgery appears to be indicated after proper clinical observation.

\section{LAPROSCOPIC CHOLECYSTECTOMY IN THE PEDIATRIC POPULATION: A SINGLE CENTER EXPERIENCE}

doi:10.1136/archdischild-2012-302724.1594

MM Zeidan, KA Ibrahim, TK Pandian, CR Moir, MB Ishitani, AE Zarroug. Division of Pediatric Surgery, Mayo Clinic, Rochester, MN, USA

Background and Aims As the preferred technique for cholecystectomy in children, we aim to review our experience with laparoscopic cholecystectomy in the pediatric population to better understand the associated complications and outcomes.

Methods We performed an IRB approved, retrospective chart review of children $\leq 18$ years who underwent cholecystectomy at a single academic institution between the years 1990 and 2010.

Results Of the 325 cases of cholecystectomy, 202 (62.2\%) were performed laparoscopically. The primary indication for surgery was symptomatic cholelithiasis $(45.5 \%, \mathrm{n}=92)$. Preoperative endoscopic retrograde cholangiopancreatography (ERCP) was performed in $25(12.4 \%)$ of cases. Variations in anatomy and technical difficulties (e.g. presence of adhesions) were found in $45(22.3 \%)$ of patients. Intraoperative cholangiogram was performed in 20 (9.9\%) and concomitant splenectomy was undertaken in $16(7.9 \%)$ cases. Only $8(4 \%)$ of cases were converted to an open fashion, all due to a lack of anatomical clarity. There were zero common bile duct injuries; however spillage of bile was present in $12(5.9 \%)$ patients. Postoperative complications including wound infection 4, retained stones 4, abdominal abscess 1 , and biloma 0 , totalled 9 patients (4.5\%). Median operative time was 117.5 minutes. Median postoperative hospital stay was 1 day and 19 (9.4\%) patients had recurrence of abdominal pain without associated pathology. Three patients $(1.5 \%)$ required postoperative ERCP. In this cohort, average follow-up was 54 months.

Abstract 1594 Table 1 Patient Characteristics

\begin{tabular}{lcccc}
\hline & $\mathbf{n}$ & $\%$ & Mean & Range \\
\hline Age (months) & & & 163.2 & $6-216$ \\
BMI (kg/m2) & & & 23.4 & $12.9-47.6$ \\
Gender (Male) & 56 & 27.7 & & \\
Gender (Female) & 146 & 72.3 & & \\
Presence of Comorbidities & 52 & 25.7 & & \\
Previous Surgical History (Abdominal) & 25 & 12.4 & & \\
Previous Surgical History (Other) & 32 & 15.8 & & \\
Admission Total Bilirubin (mg/dL) & & & 1.5 & $0.2-22.8$ \\
Admission Amylase (U/L) & & & 94.4 & $18-1184$ \\
\hline
\end{tabular}

Conclusion Laparoscopic cholecystectomy in pediatric population results in short postoperative hospital stays and has low complication rates. In our experience, it also leads to relatively high symptom relief.

\section{5 \\ POSTNATAL MANAGEMENT FOR PRENATALLY DIAGNOSED BILIARY CYSTIC MALFORMATIONS (BCM)}

doi:10.1136/archdischild-2012-302724.1595

T Okada, S Honda, H Miyagi, A Taketomi. Department of Gastroenterological Surgery I, Hokkaido University Graduate School of Medicine, Sapporo, Japan

Background and Aims The aim of this study was to determine an appropriate postnatal management plan for prenatally diagnosed BCM including cystic biliary atresia (BA) and choledochal cyst (CC). Methods From 2002 to 2011, a total of 27 consecutive children with CC were treated at our institute. Eight of our 27 patients with CC were diagnosed prenatally and examined clinically. Of these 8 patients, 2 (Group A) underwent delayed primary definitive surgery after percutaneous transhepatic cholangiodrainage (PTCD), 2 (Group B) underwent early definitive surgery in the neonatal period, and 4 (Group C) underwent delayed primary definitive surgery without PTCD in early infancy. Prenatally diagnosed cystic BA was consisted with 2 of patients with type 1 cystic BA (Group D).

Results The operation was difficult for adhesion in Group A. The diameter of the anastomosis in the hepaticojejunostomy was small and anastomotic leakage occurred in one of Group B. In all 2 patients, BA was recognized as final diagnosis at laparotomy and a hepaticojejunostomy was carried out because of correctable type. The pathological findings of liver biopsy revealed slight fibrosis of Glisson's sheath in 6 of 8 CC patients. Severe liver fibrosis presented in one of two in cystic BA patients.

Conclusion In symptomatic CC patients, PTCD appears to be indicated only under certain circumstances, and delayed primary definitive surgery should be performed as early as possible thereafter. Clinicians need to be aware of cystic BA and how to distinguish it from CC to avoid inadequate primary surgical intervention.

\section{OUTCOME OF COCHLEAR IMPLANTATION IN POST-MENINGITIS DEAF CHILDREN}

doi:10.1136/archdischild-2012-302724.1596

${ }^{1} \mathrm{~S}$ Amirsalari, ${ }^{2} \mathrm{~A}$ Saburi, ${ }^{3} \mathrm{H}$ Hasanalifard, ${ }^{3} \mathrm{M}$ Ajaluiyan. ${ }^{1}$ Pediatrics Department; ${ }^{2}$ Chemical Injury Research Center, ${ }^{3}$ Cochlear Implantation Research Center, Baqiyatallah University of Medical Sciences, Tehran, Iran

Background Bacterial meningitis is the most common cause of secondary sensori-neural hearing loss in pediatrics. Due to concomitant neurological squeal such as seizure, visual impairment and hydrocephalus the successful outcome of cochlear implantation is doubtful. The aim of this survey is assessment of cochlear implantation outcomes in post meningitis deaf children.

Methods Patients who were implanted at Baqiyatallah Cochlear Implant Center, during the years 2008-2010 due to post meningitis deaf childrenwere enrolled. the intraoperatively and Postoperative auditory and speech abilities were explored and compared.

Results Two hundred eighty-four children with hearing loss were evaluated and eight children who were diagnosed as Post Meningitis Deafness were enrolled. The mean age of children at the meningitis diagnosis was $15.75 \pm 6.77$ months and the mean age at cochlear implantation was $31.12 \pm 1.27$ months. Electrode insertion in 6 out of eight patients was complete but 2 children required cochlear drillout and in one child short electrode was used. The survey shows that auditory and language skills improved as well as expected. Improvement of auditory and speech abilities after 6 months was statistically significant (P.value<0.05).

Conclusions It seems that cochlear implantation outcome in post meningitis deaf children is not the same as non meningitis deaf children but the cochlear implantation is the only and in most cases the best way of helping these children, particularly if the gap time between deafness and surgery is minimized and the ossification is 